\title{
PREVALENCE OF CANDIDA ALBICANS IN CHRONIC PERIODONTITIS PATIENTS
}

\author{
Pl. Sasikumar1, Srihari J'2, Koshy Chitresan ${ }^{3}$, Arun Maradi ${ }^{4}$, Praveen Krishna ${ }^{5}$ \\ 1 Postgraduate Student, Department of Periodontics, Sri Ramakrishna Dental College, Coimbatore. \\ 2 Professor and Guide, Department of Periodontics, Sri Ramakrishna Dental College, Coimbatore. \\ 3 Professor and HOD, Department of Periodontics, Sri Ramakrishna Dental College, Coimbatore. \\ ${ }^{4}$ Reader, Department of Periodontics, Sri Ramakrishna Dental College, Coimbatore. \\ ${ }^{5}$ Reader, Department of Periodontics, Sri Ramakrishna Dental College, Coimbatore.
}

\section{ABSTRACT}

\section{BACKGROUND}

Chronic periodontitis is defined as "an infectious disease resulting in inflammation within the supporting tissues of the teeth and progressive attachment loss and bone loss." Chronic periodontitis is associated with a widely diverse and complex subgingival microbiota encompassing both Gram-positive and Gram-negative bacteria, facultative and anaerobic organisms, viruses and yeasts. More than 500 bacterial strains have been recovered from the subgingival plaque. Plaque also comprises of fungal species like Candida albicans, Candida glabrata, Candida tropicalis, Candida krusei, and Candida dubliniensis. Most of these strains are commensals and some are potential opportunistic pathogens.

The objective of the study is to examine the prevalence of Candida albicans (dimorphic fungus) in subgingival plaque samples of patients with chronic periodontitis.

\section{MATERIALS AND METHODS}

It's a cross-sectional study of 108 chronic periodontitis patients, their age ranged from 30 to 55 years. Clinical parameters like plaque index (PII), probing depth (PD), and clinical attachment level (CAL) were measured. We also observed the smokers from the analysed patients. Suitable microbiological media was used to culture C. albicans from clinical plaque samples. Data analysed with suitable statistical methods.

\section{RESULTS}

Clinical parameters of PII, PD, and CAL, disease severity and sex revealed no significant relation between $C$. albicans and periodontitis patients, while smoking of individuals significantly correlated with the presence of the C. albicans.

\section{CONCLUSION}

Although C. albicans infection occurred in chronic periodontitis patients, smokers were at higher risk for $C$. albicans infection than non-smokers.

\section{KEYWORDS}

C. albicans, Chronic Periodontitis, Smoking, Sex.

HOW TO CITE THIS ARTICLE: Sasikumar PL, Srihari J, Chitresan K, et al. Prevalence of Candida albicans in chronic periodontitis patients. J. Evolution Med. Dent. Sci. 2017;6(87):6056-6060, DOI: 10.14260/jemds/2017/1315

\begin{abstract}
BACKGROUND
Chronic periodontitis is defined as "an infectious disease resulting in inflammation within the supporting tissues of the teeth and progressive attachment loss and bone loss".[1] Chronic periodontitis is associated with a widely diverse and complex subgingival microbiota encompassing both Grampositive and Gram-negative bacteria, facultative and anaerobic organisms, viruses and yeasts. More than 500 bacterial strains have been recovered from the subgingival plaque.[2] Plaque also comprises of fungal species like Candida albicans, Candida glabrata, Candida tropicalis, Candida krusei, and Candida dubliniensis. Most of these strains are commensals and some are potential opportunistic pathogens.
\end{abstract}

'Financial or Other Competing Interest': None.

Submission 29-09-2016, Peer Review 17-10-2017,

Acceptance 25-10-2017, Published 30-10-2017.

Corresponding Author:

Dr. Pl. Sasikumar,

Department of Periodontics,

Sri Ramakrishna Dental College,

SNR College Road, Coimbatore, Tamilnadu.

E-mail: drsasikumarpl@gmail.com

DOI: $10.14260 /$ jemds/2017/1315
Candida species are commensal yeasts and opportunistic pathogens that reside on the mucosal surfaces and can cause oropharyngeal infections. It occurs usually in the immunocompromised individuals with endocrinal disorders, blood diseases and with longterm use of broad spectrum antibiotic therapy.[3] The possible relevant factors for Candida species colonisation are nutrition, bacterial interaction and the presence of specific antibodies like IgA and IgG in saliva.[4] In healthy oral carriers, Candida species typically resides on the tongue, palate, buccal mucosa and in the saliva.[5]

C. albicans is the most prevalent yeast of oral microbiota. It constitutes $60 \%$ to $70 \%$ of total isolates of this genus, but other Candida species including Candida glabrata, Candida tropicalis, Candida parapsilosis, Candida krusei, Candida dubliniensis, Candida glabrata, Candida kefyr, Candida lusitaniae and Candida viswanathii are also found. [4]

Yeasts, especially $C$. albicans, are recovered not only from the oral mucosae, but also in other oral sites such as pulp chamber, carious lesions and periodontal pockets.[6] At the subgingival sites, there was an increase in colonisation with 
Candida species in chronic periodontitis and aggressive periodontitis than the subjects with healthy periodontium.[7]

The Candida species have virulence factors that facilitate colonisation and proliferation in the oral mucosa and possibly in periodontal pockets. These fungal organisms can co-aggregate with bacteria in dental biofilm and adhere to epithelial cells. These interactions, which are associated with their capacity to invade gingival connective tissue, may be important in microbial colonisation that contributes to progression of oral diseases.[8]

The objective of the present study was to examine the prevalence of Candida albicans among chronic periodontitis patients and to assess the relationship between smoking and C. albicans infection.

\section{MATERIALS AND METHODS}

It's a prevalence study for a period of three months, the daily outpatients of Sri Ramakrishna Dental College \& Hospital, Coimbatore, Tamil Nadu state, India having chronic periodontitis were included in this study. All patients were carefully analysed, and specific points related to study were noted. The study was approved by the ethical committee of the institution. The inclusion criteria include age group between 30 and 55 years. Patients who had not received any dental treatment for the past 6 months, without any systemic complications and patients with chronic periodontitis, and any systemic and immunocompromised diseases receiving any drugs for the past 6 months were excluded from this investigation.

On each clinical examination, the following clinical parameters were calculated by Plaque Index (PII), Conventional probing depth (PD) and Clinical Attachment Loss (CAL). Subgingival plaque samples were collected from each patient with the help of a sterile Gracey curette either in buccal aspects of molar teeth or one more teeth in the deepest pockets. Collected samples were placed in a sterile container which consists of phosphate buffered saline in Eppendorf tube as a transport medium and was sent to the laboratory for the culturing of C. albicans. Culture was performed in Sabouraud's Dextrose Agar (SDA) incubated at $37^{\circ} \mathrm{C}$ for 2-3 days. C albicans growth on Sabouraud's Dextrose Agar produces creamy, moist, yeast-like colonies in a streak like pattern. Yeast colonies growing on each Sabouraud's Dextrose Agar were re-suspended and $10 \mu \mathrm{L}$ of suspension solution was used to inoculate plates with CHROM agar medium. Inoculated plates were incubated at $37^{\circ} \mathrm{C}$ and read for up to 7 days. Plates were observed for fungal growth using morphology and colour to determine the presence of yeasts. $C$. albicans were identified by the production of green coloured colonies, respectively.

Samples were Gram stained, which indicate Grampositive budding yeast cells with pseudohyphae, and Gramnegative pus cells and bacilli. Small portion of an isolated colony was suspended in a test tube containing $0.5 \mathrm{~mL}$ of human serum then incubated at $37^{\circ} \mathrm{C}$ for 2 hours, then examined microscopically at 30-minute intervals up to 2 hours for the presence of germ tube confirmatory test for $C$. albicans.

\section{Study Design}

Cross-sectional Study.

\section{Statistical Analysis}

Chi-square test done by the Software SPSS Version 13.

\section{RESULTS}

A total of 108 patients were analysed, which include 69 males and 39 females. The age group of the patients ranged between 30 and 55 years, the average was 42.9 (Table 1). The CAL measured from the CEJ to the base of the periodontal pocket using Williams periodontal probe, analysed as mild, moderate and severe chronic periodontitis based on the CAL. Among the study subjects, males had CAL with $4.5 \pm 1.1 \mathrm{~mm}$ whereas females had $4.4 \pm 1.1 \mathrm{~mm}$. Among 69 males analysed, 5 patients had mild, 26 patients had moderate and 38 patients had severe chronic periodontitis. In females, 2 patients with mild, 18 patients with moderate and 19 patients with severe chronic periodontitis were observed (Table 2, Figure 1). Comparing the severity of diseases among the sexes showed no statistical significant differences. (Table 3 , Figure 2)

In the present study, when correlating the sexes with severity of chronic periodontitis and C. albicans, none of the male and female counterparts with mild chronic periodontitis tested positive for C. albicans. Among moderate groups of chronic periodontitis, 5 males (19\%) and 2 females (11\%) were positive for $C$. albicans whereas severe chronic periodontitis with 9 males (23\%) and 4 females (21\%) were positive for $C$. albicans. The results showed no significant differences between sexes and severity of disease to the presence of $C$. albicans (Table 4, Figure 3).

The comparison of smokers and nonsmokers to the exposure of $C$. albicans infection- none of the subjects with mild chronic periodontitis tested positive for C. albicans. Among the smokers, 3 subjects (33.3\%) with moderate chronic periodontitis and 6 subjects (66\%) with severe chronic periodontitis tested positive for C. albicans. Among nonsmokers, 4 subjects (36.3\%) with moderate chronic periodontitis and 7 subjects (63\%) with severe chronic periodontitis tested positive for $C$. albicans. The result revealed that there is no statistically significant difference $(p$ $=0.888$ ) between smoking and $C$. albicans positive individuals with severity of chronic periodontitis (Table 5, Figure 4).

\begin{tabular}{|c|c|c|}
\hline Sex & $\mathbf{N}$ & Mean \pm SD \\
\hline Male & 69 & $43.3 \pm 7.3$ \\
\hline Female & 39 & $42.03 \pm 6.3$ \\
\hline Total & $\mathbf{1 0 8}$ & $\mathbf{4 2 . 9} \pm \mathbf{6 . 9 8}$ \\
\hline \multicolumn{2}{|c|}{ Table 1. Age Characteristics of the Sample } \\
\hline
\end{tabular}

\begin{tabular}{|c|c|c|c|}
\hline $\begin{array}{c}\text { Chronic } \\
\text { Periodontitis }\end{array}$ & N & $\begin{array}{c}\text { Male } \\
\text { Mean } \pm \text { SD }\end{array}$ & $\begin{array}{c}\text { Female } \\
\text { Mean } \pm \text { SD }\end{array}$ \\
\hline Mild & 7 & $2.348 \pm 0.642$ & $2.221 \pm 0.566$ \\
\hline Moderate & 44 & $3.895 \pm 0.876$ & $3.356 \pm 0.942$ \\
\hline Severe & 57 & $5.486 \pm 0.422$ & $5.433 \pm 0.141$ \\
\hline Total & $\mathbf{1 0 8}$ & $\mathbf{4 . 4 7 0} \pm 1.067$ & $\mathbf{4 . 3 9 5} \pm 1.056$ \\
\hline Table 2. Clinical Attachment Level with Severity of Chronic \\
Periodontitis \\
\hline
\end{tabular}




\begin{tabular}{|c|c|c|c|c|c|c|c|}
\hline $\begin{array}{c}\text { Chronic } \\
\text { Perio- } \\
\text { dontitis }\end{array}$ & \multicolumn{4}{|c|}{ Mex } & \multicolumn{2}{|c|}{ Total } & \multirow{2}{*}{ p Value } \\
\cline { 2 - 7 } & Count & $\mathbf{\%}$ & Count & $\mathbf{\%}$ & Count & $\%$ & \\
\hline Mild & 5 & $7.20 \%$ & 2 & $5.10 \%$ & 7 & $6.50 \%$ & \\
\hline $\begin{array}{c}\text { Mode } \\
\text { rate }\end{array}$ & 26 & $37.70 \%$ & 18 & $46.20 \%$ & 44 & $40.70 \%$ & \multirow{2}{*}{0.670} \\
\cline { 1 - 7 } Severe & 38 & $55.10 \%$ & 19 & $48.70 \%$ & 57 & $52.80 \%$ & \\
\cline { 1 - 6 } Total & $\mathbf{6 9}$ & $\mathbf{1 0 0 . 0 0}$ & $\mathbf{3 9}$ & $\mathbf{1 0 0 . 0 0}$ & $\mathbf{1 0 8}$ & $\mathbf{1 0 0 . 0 0}$ & \\
\hline \multicolumn{7}{|c|}{ Table 3. Severity of Chronic Periodontitis- Gender wise } \\
\hline
\end{tabular}

$\chi^{2}=0.000(p$ value $>0.05$ not significant $) \chi^{2}$ - chi square test

\begin{tabular}{|c|c|c|c|c|c|c|c|c|c|c|}
\hline \multirow{2}{*}{ 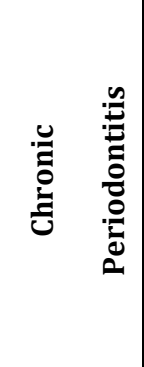 } & \multicolumn{3}{|c|}{ 䒿 } & \multicolumn{3}{|c|}{ 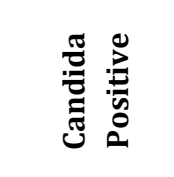 } & \multicolumn{3}{|c|}{ 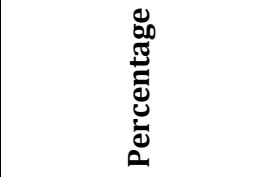 } & \multirow{3}{*}{$\begin{array}{l}\frac{0}{\Xi} \\
\frac{\pi}{\pi} \\
2 \\
\end{array}$} \\
\hline & $\frac{0}{\sum \pi}$ & 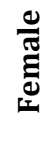 & 胥 & $\frac{0}{\sum^{\pi}}$ & & 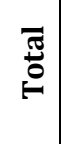 & $\frac{0}{\frac{\pi}{\pi}}$ & 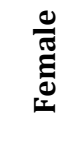 & 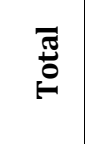 & \\
\hline Mild & 5 & 2 & 7 & 0 & 0 & 0 & $0 \%$ & $0 \%$ & $0 \%$ & \\
\hline Moderate & 26 & 18 & 44 & 5 & 2 & 7 & $19 \%$ & $11 \%$ & $14 \%$ & \\
\hline \multirow{2}{*}{ Severe } & 3 & 1 & \multirow{2}{*}{57} & \multirow{2}{*}{9} & \multirow{2}{*}{4} & 1 & 23 & 21 & 22 & \\
\hline & 8 & 9 & & & & 3 & $\%$ & $\%$ & $\%$ & \\
\hline Total & 69 & 39 & 108 & 14 & 6 & 20 & $20 \%$ & $15 \%$ & $18 \%$ & \\
\hline
\end{tabular}

$\chi^{2}=0.000(p$ value $>0.05$ not significant $) \chi^{2}$ - chi square test

\begin{tabular}{|c|c|c|c|c|c|c|}
\hline \multirow{2}{*}{$\begin{array}{c}\text { Smoking } \\
\text { Habit }\end{array}$} & $\begin{array}{c}\text { Candida } \\
\text { Positive } \\
(+)\end{array}$ & & \multicolumn{2}{|c|}{ Chronic Periodontitis } & \multirow{2}{p}{} \\
\cline { 3 - 6 } Smoker & 9 & Count & 0 & 3 & 6 & \\
\cline { 3 - 6 } & $\%$ & $0.00 \%$ & $33.30 \%$ & $66.70 \%$ & \multirow{2}{*}{0.888} \\
\cline { 3 - 6 } $\begin{array}{c}\text { Non- } \\
\text { smoker }\end{array}$ & 11 & Count & 0 & 4 & 7 & \\
\cline { 3 - 6 } & $\%$ & $0.00 \%$ & $36.36 \%$ & $63.63 \%$ & \\
\hline Table 5. Smoking Habits of Candida albicans Positive \\
Cases with Chronic Periodontitis \\
\hline
\end{tabular}

\section{$\chi^{2}=0.000(p$ value $>0.05$ not significant $) \chi^{2}$ - chi square test}

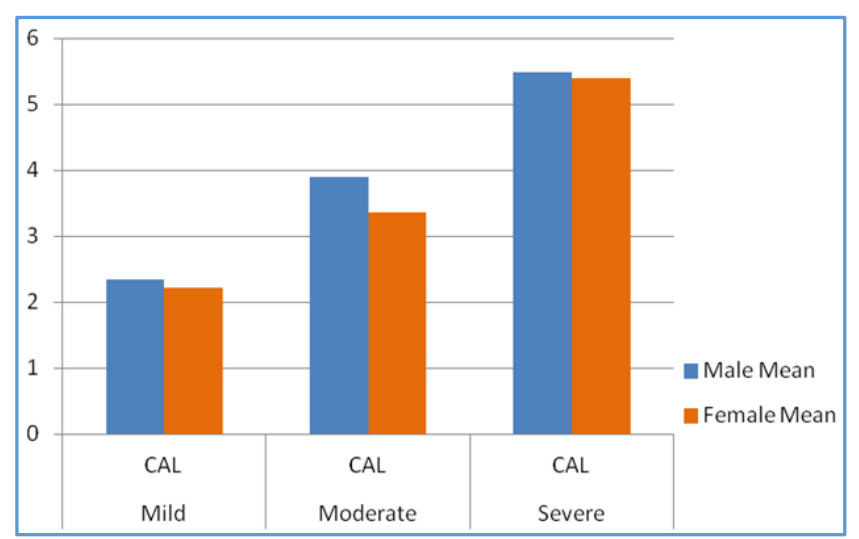

Figure 1. Clinical Attachment Level with Severity of Chronic Periodontitis

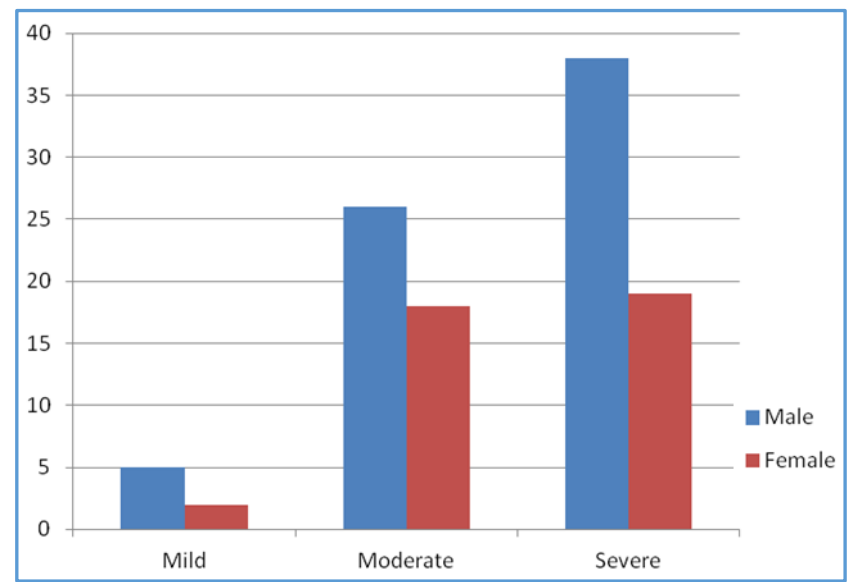

Figure 2. Severity of Chronic Periodontitis- Gender wise

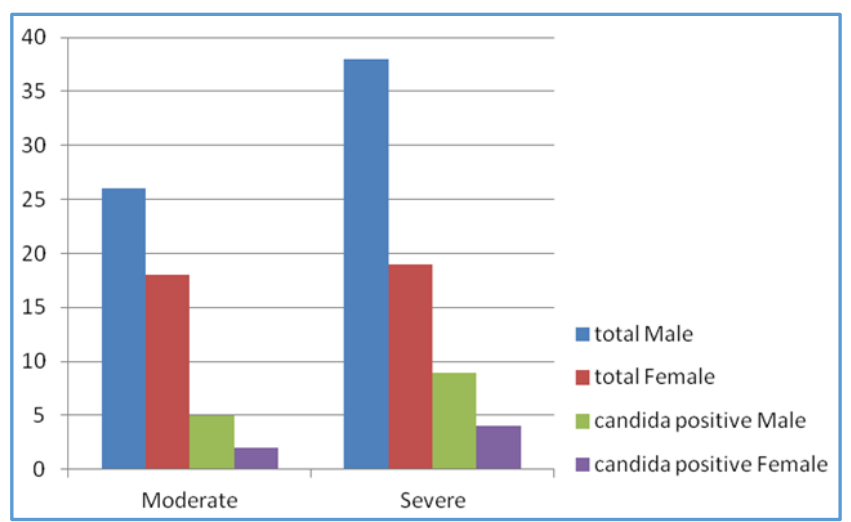

Figure 3. Severity of Chronic Periodontitis and Candida albicans Positive Cases- Gender wise

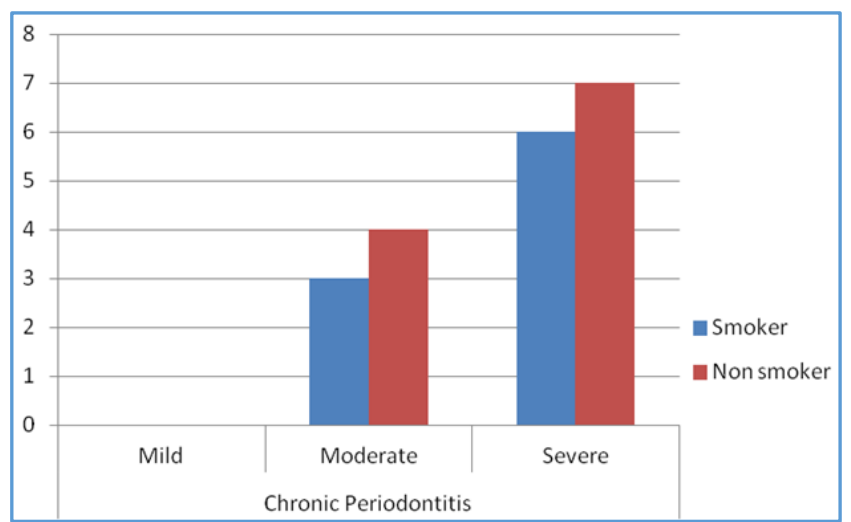

Figure 4. Smoking Habits of Candida albicans Positive Cases with Chronic Periodontitis

\section{DISCUSSION}

C. albicans may aid the plaque microorganisms in evading the host defence mechanism as it is typically found on the outer layers of the plaque and has also been observed in deep periodontal tissues.[9] $C$. albicans was identified in the subgingival sites of patients with severe chronic periodontitis, suggesting that the advanced form of chronic periodontitis was associated with a more complex yeast community residing in the deep pockets.[10] Severe periodontal disease may be one of the causes for immunosuppression, which leads to colonisation of this opportunistic pathogen.[9] Though Candida can appear in pseudohyphae or yeast forms, the pseudohyphae is the one 
found more common in tissues, whereas the yeast forms are found on epithelial surfaces. Hyphae have the ability to penetrate host tissue and are hence important in the disease process. The gingival pocket and gingival crevicular fluid provide a favourable environment for the germination and growth of these hyphae.[9] C. albicans can secrete proteinases capable of degrading major extracellular matrices and basement membrane components that cause destructive inflammation of the underlying periodontal tissues.[11,12,13]

Based on these observations, in the current study, subgingival plaque samples were collected from the deepest periodontal sites using sterile curettes from 108 subjects. This comprehensive sampling regimen was carried out in order to get an accurate representation of C. albicans prevalent in the periodontal pockets of patients with chronic periodontitis. The clinical parameters were assessed using plaque index, probing pocket depth and clinical attachment level. Sabouraud's dextrose agar and the CHROM agar media were used to culture $C$. albicans from clinical samples in order to exhibit characteristic colony colours and for the detection of separate Candida species.

Based on the amount of clinical attachment loss, subjects were classified into mild, moderate and severe chronic periodontitis.[14] In the current study, CAL for mild, moderate and severe chronic periodontitis for male subjects was 2.348 $\pm 0.64,3.895 \pm 0.876$, and $5.486 \pm 0.422$ respectively and for female subjects $2.221 \pm 0.566,3.356 \pm 0.942$ and $5.433 \pm$ 0.141 respectively. In the present study, the prevalence of $C$. albicans was $18.5 \%$. This was in accordance with studies done by Reynaud et al.[15] in Norway who demonstrated prevalence of $17.5 \%$ in 128 subjects that were studied and Canbarro et al[10] who reported a prevalence of $17 \%$ in the Brazilian population. Among 20 subjects who were positive for C. albicans, $14(20.3 \%)$ were male and $6(15.4 \%)$ were female, which showed that the prevalence of $C$. albicans did not show any statistically significant $(\mathrm{p}=0.528)$ difference between males and females. This result was in accordance with the studies done by Slots et al.[16]

In the present study, of the 7 subjects diagnosed with mild chronic periodontitis, none were positive for $C$. albicans. $14 \%$ of the subjects with moderate chronic periodontitis and $22 \%$ of the subjects with severe chronic periodontitis were $C$. albicans positive. This result was in contrast to the study done by Canbarro et al[10] in Brazilian population, where $C$. albicans was present in $47 \%$ subjects with moderate chronic periodontitis though similar results were found in $17 \%$ of the C. albicans positive patients with severe chronic periodontitis. These differences could be attributed to the geographical and ethnic differences among the subjects in both the studies, and also the limited sample size in this study.

In the present study among the subjects who tested positive, C. albicans was significantly higher in smokers (31\%) than in the non-smokers $(13.0 \%)$ with $p=0.042$, showing that tobacco smoking increases the prevalence of $C$. albicans. This result was in accordance with the study done by Keten et al.[17] in Turkey which showed that $30 \%$ of the study samples were $C$. albicans positive in smokers compared to $18.3 \%$ in nonsmokers.

However, other studies have found that tobacco smoking did not have an influence on oral colonisation with $C$. albicans. Oliver \& Shillitoe ${ }^{[18]}$ demonstrated that $C$. albicans was prevalent in $35 \%$ of the smokers and in $35 \%$ of the nonsmokers. Darwazeh et al.[19] isolated C. albicans from 84\% of the smokers and $74 \%$ of the nonsmokers and they found no significant association between smoking habits and $C$. albicans.

Higher candidal count observed among the smokers may be attributed to the aromatic hydrocarbons in tobacco, which have been shown to act as nutrients to the yeast cells. Smoking may indirectly increase the level of salivary glucose, which enhances yeast growth. Also, smoking can depress the activity of oral leucocytes and other nonspecific immune defences.[19]

To the best of our knowledge, this is the first study to elaborate the prevalence of $C$. albicans, in mild, moderate and severe chronic periodontitis among smokers and nonsmokers. Among the 9 smokers who tested positive for $C$. albicans, 3 subjects had moderate chronic periodontitis $(33.3 \%)$ and 6 subjects had severe chronic periodontitis (66.7\%). Among the 11 nonsmokers, who tested positive for C. albicans, 4 subjects had moderate chronic periodontitis $(36.3 \%)$ and 7 subjects had severe chronic periodontitis (63.6\%).

As the sample size was small in this study, the statistical validity of the associations found was limited. Hence, large sample sizes are required to confirm the clinical findings, and further qualitative and quantitative analysis should be done to validate the results of this study.

\section{CONCLUSION}

C. albicans was present in higher amounts in the periodontal pockets of patients with severe chronic periodontitis. Smokers were at higher risk for $C$. albicans infection than non-smokers. $C$. albicans did not have a predilection for any particular sex.

\section{REFERENCES}

[1] Flemmig TF. Periodontitis. Ann Periodontol 1999;4(1):32-8.

[2] Kroes I, Lepp PW, Relman DA. Bacterial diversity within the human subgingival crevice. Proc Natl Acad Sci USA 1999;96(25):14547-52.

[3] Webb BC, Thomas CJ, Wilcox MD, et al. Candida associated denture stomatitis. Aetiology and management: a review. Part 1. Factors influencing distribution of Candida species in the oral cavity. Aust Dent J 1998;43(1):45-50.

[4] Stenderup A. Oral mycology. Acta Odontol Scand 1990;48(1):3-10.

[5] Arendorf TM, Walker DM. The prevalence and intraoral distribution of Candida albicans in man. Arch Oral Biol 1980;25(1):1-10.

[6] Dahlen G, Wikstrom M. Occurrence of enteric rods, staphylococci and Candida in subgingival samples. Oral Microbiol Immunol 1995;10(1):42-6.

[7] Urzua B, Hermosilla G, Gamonal J, et al. Yeast diversity in the oral microbiota of subjects with periodontitis: Candida albicans and Candida dubliniensis colonize the periodontal pockets. Med Mycol 2008;46(8):783-93.

[8] Sardi JC, Duque C, Mariano FS, et al. Candida spp. in periodontal disease: a brief review. J Oral Sci 2010;52(2):177-85. 
[9] Jarvensivu A, Hietanen J, Rautemaa R, et al. Candida yeasts in chronic periodontitis tissues and subgingival microbial biofilms in vivo. Oral Dis 2004;10(2):106-12.

[10] Canabarro A, Valle C, Farias MR, et al. Association of subgingival colonization of Candida albicans and other yeasts with severity of chronic periodontitis. J Periodontal Res 2013;48(4):428-32.

[11] Kaminishi H, Hagihara Y, Hayashi S, et al. Isolation and characteristics of collagenolytic enzyme produced by candida albicans. Infect and Immun 1986;53(2):312-6.

[12] El Moundi B, Rodier M, Barrault C, et al. Purification and characterization of a metallopeptidase of Candida albicans. J Med Microbiol 1995;43(4):282-8.

[13] Rodier MH, el Moudni B, Kauffmann-Lacroix C, et al. A candida albicans metallopeptidase degrades constitutive proteins of extracellular matrix. FEMS Microbiology Lett 1999;177(2):205-10.
[14] Armitage GC. Development of a classification system for periodontal diseases and conditions. Ann Periodontol 1999;4(1):1-6.

[15] Reynaud AH, Nygaard-Ostby B, Boygard GK, et al. Yeasts in periodontal pockets. J Clin Periodontol 2001;28(9):860-4.

[16] Slots J, Rams TE, Listgarten MA. Yeasts, enteric rods and pseudomonads in the subgingival flora of severe adult periodontitis. Oral Microbiol Immunol 1988;3(2):47-52.

[17] Keten HS, Keten D, Ucer H, et al. Prevalence of oral Candida carriage and Candida species among cigarette and maras powder users. Int J Clin Exp Med 2015;8(6):9847-54.

[18] Oliver DE, Shillitoe EJ. Effects of smoking on the prevalence and intraoral distribution of Candida albicans. J Oral Pathol 1984;13(3):265-70.

[19] Darwazeh AM, Hammad MM, Al-Jamaei AA. The relationship between oral hygiene and oral colonization with Candida species in healthy adult subjects. Int J Dent Hygiene 2010;8(2):128-33. 